\title{
Parallel Visual Data Restoration on Multi-GPGPUs using Stencil-Reduce Pattern
}

\author{
Marco Aldinucci ${ }^{1}$, Guilherme Peretti Pezzi ${ }^{1}$, Maurizio Drocco ${ }^{1}$, Concetto Spampinato ${ }^{2}$, Massimo Torquati ${ }^{3}$ \\ ${ }^{1}$ Computer Science Dept., University of Torino, Italy \\ ${ }^{2}$ Dept. of Electrical, Electronics and Computer Engineering, University of Catania, Italy \\ ${ }^{3}$ Computer Science Dept., University of Pisa, Italy
}

\begin{abstract}
In this paper, a highly effective parallel filter for visual data restoration is presented. The filter is designed following a skeletal approach, using a newly proposed stencil-reduce, and has been implemented by way of the FastFlow parallel programming library. As a result of its high-level design, it is possible to run the filter seamlessly on a multicore machine, on multi-GPGPUs, or on both. The design and implementation of the filter are discussed, and an experimental evaluation is presented.
\end{abstract}

\section{Index Terms}

Impulsive noise, Gaussian noise, Image restoration, Image filtering, GPGPUs, Parallel patterns, Skeletons, Structured parallel programming, Iterative Stencil, stencil-reduce, MapReduce

\section{INTRODUCTION}

In the last decade, the advances in camera technology and the reduction of costs of memory storage have led to a proliferation of visual data content in the form of images and videos, e.g. 72 hours of video are uploaded to YouTube every minute. This visual data deluge combined to the ever increasing computing power available from off-the-shelf processors has opened new frontiers in the research on automatic video and image analysis with the goal to push such problems into consumer applications. This latter aspect demands for real-time analysis of digital images and videos, i.e. effective strategies for reducing the processing times and resources needed by the existing approaches from image pre-processing to visual content extraction and understanding.

Image restoration is a key module of any machine vision system and aims at removing noise and restoring the original visual content: noise often generated by image sensor failures. Variational methods are well known for their effectiveness in image restoration [1], [2], but they cannot be employed for real-time video and image analysis because of their high computational cost, due to function minimisation over all the image pixels, and complexity of tuning. An efficient image restoration approach for images corrupted by Salt and Pepper noise based on variational methods is described in [3]. In detail, a two-phase parallel image restoration schema running on both multicore and GPGPUs is described: a) in the first step, an adaptive median filter is employed for detecting noisy pixels, while b) in the second step, a regularisation procedure is iterated until the noisy pixels are replaced with values able to preserve image edges and details. The restoration method is implemented according to a high-level pattern-based approach (a.k.a. skeletal approach [4]), and deployed as a sequence of detect and restoration stages that are defined according to the map parallel paradigm and the map-reduce parallel paradigm, respectively.

This paper extends the previous work in the following directions:

- A newly designed stencil-reduce pattern that allows offloading iterative and stencil-based computation to GPGPU devices is presented and tested on image restoration applications;

- The restoration schema, previously proposed, is extended in order to deal also with Gaussian noise and not only with impulse noise;

- The restoration schema is, finally, applied to video streams and is expressed using pipeline parallelism between the two stages.

A first prototypal implementation working on video streams has been proposed in [5], which is based on hand-written OpenCL kernels for GPGPUs offloading, while in this paper a higher level approach is proposed, based on the recently proposed stencil-reduce pattern from the FastFlow framework, where all the memory management and offloading calls are hidden from the programmer.

The remainder of the paper is as follows: firstly the background is presented, including the state of the art on variational based image restoration and an introduction about algorithmic skeleton solutions and the FastFlow framework; secondly, the new

Corresponding author:

Marco Aldinucci, Computer Science Dept., University of Torino, Corso Svizzera 185, 10149 Torino, Italy

e-mail: aldinuc@di.unito.it 
iterative stencil-reduce pattern it is presented along with the design of the parallel restoration process; thirdly, experimental evaluation of quality and performance of the proposed parallel restoration algorithm are presented; eventually, concluding remarks and future works are discussed.

\section{BACKGROUND}

In this section the related work on image restoration process will be first introduced. Then, an overview of state-of-the-art structured parallel programming frameworks is given, together with an introduction to the FastFlow framework.

\section{Variational Image Restoration: Related Work}

In the past fifteen years, a large number of methods have been proposed for effective image filtering and restoration with a particular emphasis to impulse noise [6]. Most of these methods employ order statistic filters that exploit the rank-order information of an appropriate set of noisy input pixels. The median filter is the most popular non-linear filter for removing impulse noise, because of its restoration effectiveness [7] and computational efficiency [8]. However, the performance of median filter is unsatisfactory both in detail preserving and in suppressing signal-dependent noise with noise over 50\% [9].

An effective impulse noise filtering should be able to remove as much noise as possible, while preserving high-frequency image components. This issue has generally been addressed by methods based on median filter [10], [11]. For example, the Center-Weighted Median (CWM) filter weights more the central value of a kernel window in order to make it more robust against outliers, thus preserving more details than the median filter. This is obtained at the expense of less noise suppression. The Adaptive Center-Weighted Median (ACWM) [11], instead, exploits local features computed on image patches to calculate weights. Typically, median based filters are used invariantly across the whole images to remove noise, thus smearing image details [12] since it also changes the values of uncorrupted pixels.

To achieve a good compromise between image-detail preservation and noise reduction, a noisy pixel detector is generally adopted to identify corrupted pixels, which are then iteratively restored. An example is the Multi-State Median Filter (MSMF) [13], which consists of a two-part noisy pixel detection stage and a correction stage by applying the median filter. The detection is based on the concept of homogeneity level, which defines a pixel as noisy according to the intensity differences between that pixel and its neighbours (i.e., according to its homogeneity to the neighbourhood). The second part of the detection stage re-analyses the noisy pixels using a $5 \times 5$ observation window. The main shortcoming of these methods is that they introduce undesirable distortions into the details and texture of the input image during the noise removal process. Moreover, their performance is strongly influenced by several factors, such as noise density estimate, weighting factors, impulse detection thresholds, etc. that are often heuristically determined.

The problem of image restoration for edge preserving is an inverse problem and it has been tackled mainly with variational based approaches [1]. This type of methods identify the restored image by minimising an energy function based on a datafidelity term, related to the input noise, and a regularisation term where a-priori knowledge about the solution is enforced. More specifically, this kind of approaches identifies the restored image $u$ through an optimisation problem in the form of:

$$
\min _{u \in N} F(u)=\alpha \int R(u)+\mu \int D(u, d)
$$

where $d$ is the image corrupted by the noise, $D(u, d)$ is a data-fidelity, which depends on the kind of noise and provides a measure of the dissimilarity between $d$ and the output image $u, R(u)$ is a regularisation term where a-priori knowledge about the solution is enforced, $\mu$ and $\alpha$ are the regularisation parameters that balance the effects of the two terms. This optimisation problem is restricted only to the noisy pixels $N$.

An example of variational approach for image restoration is the one proposed by Chan et al. in [2] which is capable of removing effectively impulse noise as high as $90 \%$. Similar approaches to Chan's method, aimed at improving the noisy detection step and at reducing the processing times, are those proposed in [14]-[18].

Over the years, a large variety of variational and regularisation methods have been also conceived (though less frequently than in case of impulse noise) to cope with blurring effects in order to recover the loss of high-frequency information entailed by blurring. An example is the Mumford-Shah regularisation term [19], which, however, does not work well in case of only Gaussian noise [20]. Moreover, most of the existing methods though being able to effectively filter and deblur an image, are extremely time-consuming (e.g., the method in [2] takes about 27 hours to restore a $320 \times 240$ image on a standard PC using a MATLAB implementation), thus making their application to real-world cases unfeasible and, therefore, demanding for effective parallelisation strategies. 


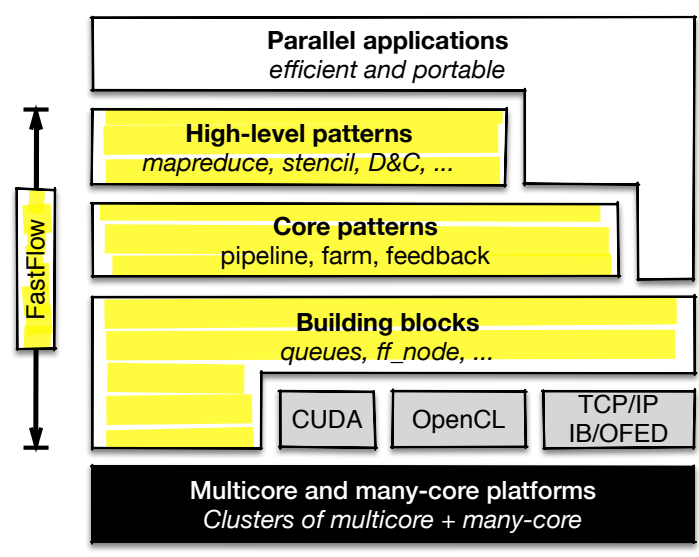

Fig. 1. Architecture of FastFlow framework.

\section{Algorithmic Skeletons}

Algorithmic skeletons have been around since the '90s as an effective means of parallel application development. An algorithmic skeleton ca be regarded as a general-purpose, parametric parallelism exploitation pattern [4]. Application programmers may instantiate skeletons (or compositions of skeletons) to encapsulate and exploit the full parallel structure of their applications. Business code may be passed as a parameter to the generic skeleton, thus turning the generic skeleton into a part of a parallel application. Programming frameworks based on algorithmic skeletons have been recently introduced to alleviate the task of the application programmer when targeting data parallel computations on heterogeneous architectures as multi-GPGPU systems.

OpenCL is a parallel API provided for GPGPU programming, which allows the users to exploit GPUs for general-purpose tasks that can be parallelised [21]. It is implemented by different hardware vendors such as Intel, AMD, and NVIDIA, making it highly portable and allowing the code written in OpenCL to be run on different graphical accelerators. It has the capability to revert to the CPU for execution when there is no GPGPU in the system. Its portability makes it suitable for Hybrid (CPU/GPGPU) or cloud-based environments. However OpenCL is quite low level, actually focusing on low-level feature management rather than high-level parallelism exploitation patterns. In Muesli [22] the programmer must explicitly indicate whether GPGPUs are to be used for data parallel skeletons. StarPU [23] is focused on handling accelerators such as GPGPUs. Graph tasks are scheduled by its run-time support on both the CPU and various accelerators provided the programmer has given a task implementation for different programming models and architectures. SkePU [24] provides programmers with GPGPU implementations of map and reduce skeletons and relies on StarPU for the execution of stream parallel skeletons (pipe and farm). MCUDA [25] is a framework to mix CPU and GPGPU programming. In MCUDA it is mandatory to define kernels for all available devices but the framework can not make any assumptions about the relative performance of the supported devices.

Moving to frameworks not specifically designed for targeting GPGPU offloading, MPI is often considered as the mainstream solution for writing efficient parallel applications [26] for heterogeneous architectures. The low-level approach advocated by MPI falls short in supporting performance portability, especially when hundreds or thousands of concurrent activities are involved and hybrid solutions have to be adapted (i.e. MPI+OpenMP). Applications must often be re-designed or manually tuned for each new platform by an expert parallel programmer. OpenMP [27] is a popular thread-based framework for multicore architectures mostly targeting data parallel programming (although it is currently being extended to incorporate stream processing). OpenMP supports, by way of language pragmas, the low-effort parallelisation of sequential programs; however, these pragmas are mainly designed to exploit loop-level data parallelism (e.g. do_independent). OpenMP does not natively support either farm or Divide\&Conquer patterns, even though they can be simulated with lower-level features. Intel Threading Building Blocks (TBB) [28] is a C++ template library, which eases the development of concurrent programs by exposing (simple) skeletons and parallel data structures used to define tasks of computations. TBB is designed as an application-level library for shared-memory programming only; furthermore it does not provide any formal definition of its own skeletons to support global optimisations of the code.

\section{The FastFlow Programming Framework}

The FastFlow parallel programming environment [29], [30] was originally designed to support efficient streaming on cachecoherent multicore platforms. It is realised as a $\mathrm{C}++$ pattern-based parallel programming framework aimed at simplifying the development of applications for (shared-memory) multicore and GPGPUs platforms. The key vision of FastFlow is that easeof-development and runtime efficiency can both be achieved by raising the abstraction level of the design phase. It provides 

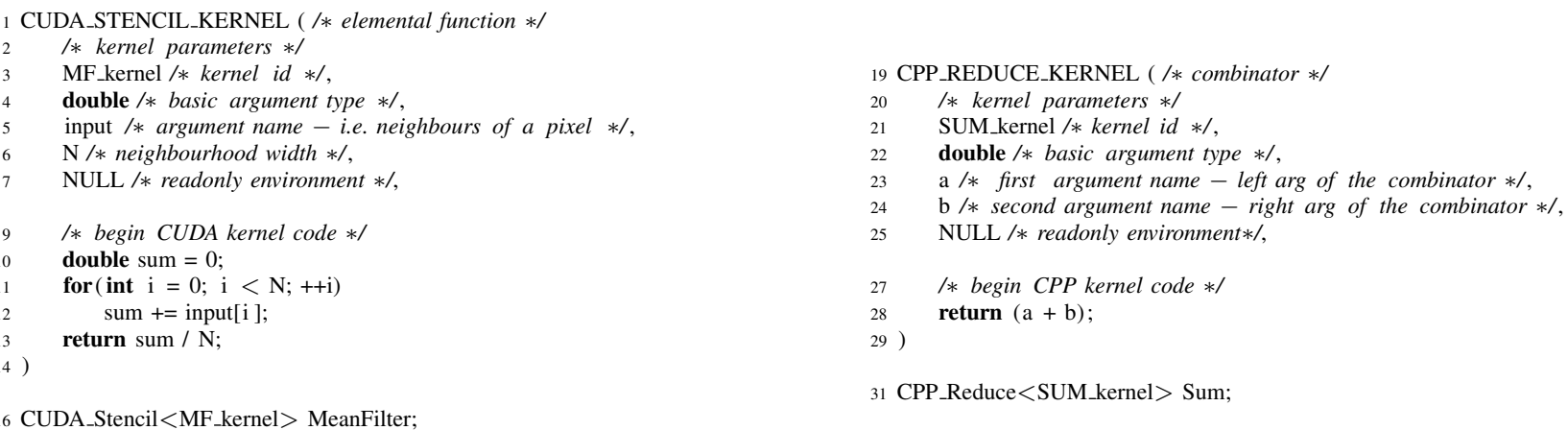

Fig. 2. 1D-convolution stencil-reduce example.

developers with a set of parallel programming patterns (aka algorithmic skeletons), in particular data-parallel patterns (such as map, stencil, reduce and their composition, and also a ParallelFor accelerator [31]). High-level patterns are implemented on top of the Core patterns level, consisting of arbitrarily nested and composed basic stream-parallel patterns (farm, pipeline and feedback).

The latest extensions of the FastFlow framework, aimed at supporting heterogeneous platforms, make it possible to easily port the application to hybrid multicore/GPGPU systems by embedding CUDA/OpenCL business code. In particular, dataparallel patterns can be run both on multicore and offloaded onto GPGPUs. In the latter case, the business code can include GPGPU-specific statements (i.e. CUDA or OpenCL statements).

At the bottom level (i.e. the Building blocks level) FastFlow CPU implementation of patterns are realised via non-blocking graphs of threads connected by way of lock-free channels [32], while the GPGPU implementation is realised by way of the CUDA/OpenCL bindings and offloading techniques [33]. The framework also takes care of memory transfers between CPU host and GPGPU device. In general, different patterns can be mapped onto different sets of cores or accelerators, thus, in principle, using the full available power of the heterogeneous platform. The architecture of FastFlow framework is reported in Fig. 1.

\section{Visual Data Restoration: A SKeleton-Based Approach}

In this work, we consider the extension of the two-phase image restoration to a two-phase video stream restoration. We advocate the high-level extension of the application working for images presented in [3] to video, and to heterogeneous platforms. We emphasise the high-level approach. In the long term, writing parallel programs that are efficient, portable, and correct must be no more onerous than writing sequential programs. To date, however, few parallel programming models have embraced much more than low-level libraries, which often require the architectural re-design of the application. This approach is unable to effectively scale to support the mainstream of software development where human productivity, total cost and time to solution are equally, if not more, important aspects.

\section{Stencil-reduce pattern}

Let map $f\left[a_{0}, a_{1}, \ldots\right]=\left[f\left(a_{0}\right), f\left(a_{1}\right), \ldots\right]$ and reduce $\oplus\left[a_{0}, a_{1}, \ldots\right]=a_{0} \oplus a_{1} \oplus \ldots$, where $f$ is the elemental function, $\oplus$ is the combinator (i.e. a binary associative operator), and $\left[a_{0}, a_{1}, \ldots\right]$ an array (e.g. the pixels of an image). These parallel paradigms have been proposed as patterns for multicore and distributed platforms, GPGPUs, and heterogeneous platforms [24], [34], [35]. Let stencil be a map, except each instance of the elemental function accesses neighbours of its input (e.g. crossshaped 4-neighborhood of a pixel), offset from its usual input [36]. They are well-known examples of data-parallel patterns; since elemental function of a map/stencil can be applied to each input element $a_{i}$ independently from each other as well as applications of the combinator to different pairs in the reduction tree of a reduce can be done independently, thus naturally inducing a parallel implementation. FastFlow framework provides constructors for these patterns, in form of C++ macros that take as input the code of the elemental function (combinator) of the map/stencil (reduce) patterns, together with an eventual read-only structure (i.e. the environment) that can be regarded, under a pure functional semantics perspective, as a function parameter. The language for the kernel code implementing the elemental function, which is actually the business code of the application, has clearly to be platform-specific (i.e. CUDA/OpenCL for GPGPUs, C++/OpenCL for multicore CPUs), since FastFlow is a plain $\mathrm{C}++$ header-only library.

In this work we use stencil-reduce pattern to realise iterative applications of non-linear filters followed by residual reduction. In order to show the usage of the stencil-reduce pattern in FastFlow data-parallel programming model, here we present a simpler case study. 
In order to use the proposed pattern, the programmer has to write three macros:

1) that defines the parameters and the elemental CUDA function that will be applied to each element using the desired stencil shape;

2) that defines the CUDA combinator function that will be applied after the map phase;

3) the stencil-reduce macro that takes as arguments the user-defined datatype that will encapsulate all needed data and macros as described in 1) and 2).

The user can additionally choose the convergence criteria that will decide when the iterative mechanism should stop, based, for example, on the result obtained from the combinator function or by simply defining an upper bound to the number of iteration.

In Fig. 2 is sketched the implementation of a simple convolution filter followed by a trivial sum operator. In the stencil phase, a classic 1D-convolution filter ( $N$-neighbours MeanFilter with $N$ odd) is applied on input array $A$, thus producing output array $A^{\prime}$. In the reduce phase, the residual array $\left|A^{\prime}-A\right|$ is passed through a reduce operator (a standard Sum) which computes the sum of its elements. In the example, CUDA version of the stencil pattern is used, thus exploiting automatic GPGPU offloading.

We remark, under the ease-of-development perspective, that the user has to provide only the kernel code of the elemental function of the Mean Filter stencil-based operator and the combinator of the Sum reduce-based operator. Moreover, under the performance portability perspective, notice that the user is not required to care about the underlying platform - except provide platform-specific kernel code - since FastFlow manages platform heterogeneity during the application deployment.

\section{Two-Phase Image Restoration}

The restoration process used in this work is a two-step algorithm and consists, first, of a noisy pixel detection phase (in the following referred as Detect) where pixels which are likely to be corrupted by noise are identified and, then, of a pixel restoration phase (referred as Restore), based on variational method [1], where the set of noisy pixels are restored in order to preserve image details.

1) Detect phase: the well-known adaptive median filter is first applied to the noisy image with the goal to identify corrupted pixels. Let $\hat{y}$ be the map obtained by thresholding the difference between the output of the adaptive median filter and the noisy original image. This map has ones for candidate noisy pixels and zeros for the remaining pixels. Each pixel of the image, at this stage, is processed independently, provided the processing element can access a read-only halo of the pixel. Hence the set of noisy pixels can be defined as follows:

$$
N=\left\{(i, j) \in \mathrm{A}: \hat{y}_{i, j}=1\right\}
$$

The set of all uncorrupted pixels is $N^{c}=N \backslash A$, where $\mathrm{A}$ is the set of the all pixels and $N$ is the set of the noisy pixels.

2) Restore phase: is carried out by means of regularisation and, among all the variational functions identified by Formula 1, we adopt the following one, as it proved [1] to operate effectively for impulse noise:

$$
\begin{aligned}
& \left.F_{d}\right|_{N}(u)=\sum_{(i, j) \in N}\left[\left|u_{i, j}-d_{i, j}\right|+\frac{\beta}{2}\left(S_{1}+S_{2}\right)\right] \\
& S_{1}=\sum_{(m, n) \in V_{i, j} \cap N^{c}} 2 \cdot \varphi\left(u_{i, j}-d_{m, n}\right) \\
& S_{2}=\sum_{(m, n) \in V_{i, j} \cap N} \varphi\left(u_{i, j}-u_{m, n}\right)
\end{aligned}
$$

where $N$ represents the noisy pixels set and where $V_{i, j}$ is the set of the four closest neighbours of the pixel with coordinates $(i, j)$ of image $d$ and $u$ is the restored image. $\left.F_{d}\right|_{N}(u)$ is given by the sum of two terms: a data fidelity term which represents the deviation of restored image $u$ from the data image $d$, which is marred by noise, and a regularisation term that incorporates a function that penalises oscillations and irregularities, although does not remove high level discontinuities. The method proposed in [37] is used for functional minimisation. The regularisation term is given by the function $\varphi$ and different forms of $\varphi$ have been employed according to the type of noise affecting the image. In this paper we employed:

$$
\begin{gathered}
\varphi_{S}(t)=|t|^{\alpha} \quad 1<\alpha \leq 2 \\
\varphi_{G}(t)=\sqrt{\left(t^{2}+\alpha\right)} \quad \alpha<<1
\end{gathered}
$$

for dealing with Salt and Pepper (a specific case of impulse noise) and Gaussian noise, respectively. As previously mentioned, especially for dealing with impulse noise, many other regularisation functions have been devised in the last ten years, e.g. $\varphi_{1}(t)=1+|t| / \alpha-\log (1+|t| / \alpha)$ and $\varphi_{2}(t)=\log (\cosh (t / \alpha))$ with $\alpha>0$. 
The variational method based on minimisation is iteratively applied until a convergence is reached, thus resulting into a fixed-point procedure. Let $u^{(k)}$ be the output of the $k$-th iteration of the procedure and $\Delta^{(k)}=\left|u^{(k)}-u^{(k-1)}\right|$ the pixel-wise residual of two successive iterations of the procedure. We used the following convergence criterion:

$$
\frac{\sum_{i, j}\left|\Delta_{i, j}^{(k)}-\Delta_{i, j}^{(k-1)}\right|}{N}
$$

Notice that the computation of the convergence criterion involves three different (successive) outcomes of the procedure, since the naive version of the criterion, based on the comparison of the two most recent outcomes, results in a strongly oscillatory convergence curve.

In this work, we only consider a two-copy approach for the Restore stage, in which the procedure at the $(k)$-th iteration takes as input the output of the $(k-1)$-th iteration in a read-only manner. Although, other restoration procedures are based on a intra-iteration approach, in which the output of the algorithm over a pixel uses as input the output of the algorithm at the same iteration for the already visited neighbours. This should guarantee a faster convergence of the iterative procedure. Notice that in a sequential implementation this induces a semantics, which is dependent on the order in which pixels are visited, thus this aspect must be taken into account when designing a parallel implementation of a intra-iteration procedure. We experimented different parallelisation schemes in such scenario, from clustering pixels into non-overlapping blocks - thus respecting the sequential semantics - to a purely non-deterministic approach, in which pixels are read regardless the fact they have already been visited by the current iteration. Anyway we did not get any appreciable gain in terms of convergence speed for the proposed functional.

3) Parallel implementation: The parallel processing in the Detect phase could in theory be exploited in some data-parallel fashion (e.g. stencil-reduce pattern). However, for images of standard resolutions (up to HD quality), as those considered in the experimental section of this work, the Detect phase has a negligible execution time w.r.t. the Restore stage, so the parallelisation of the Detect phase is not taken into account.

In the Restore phase, the parallel processing is described as an iterative stencil-reduce pattern. Within a single iteration, for each couple of outlier coordinates $(i, j)$, corresponding to an outlier pixel (i.e. a pixel in the noisy set $N$, which is the outcome of the Detect phase), the stencil accesses the eight neighbour pixels (with replication padding for borders). For each outlier $(i, j)$, the procedure computes a new value as the one minimising the value of the functional $\left.F_{d}\right|_{N}(u)$ in Formula 1 . We remark that in this scenario the user has to provide only the code of the computation over a single pixel - which is actually the business code of the Restore iteration - and the framework takes care of applying the computation over all the (noisy) pixels and iterating the procedure until the convergence condition is reached. In the reduce pattern, the global convergence criterion is computed as a simple average of the three-iteration residuals, as discussed above. In this case the user has to provide only the code of the binary combinator, which is turned into a tree-shaped parallel computation by the framework.

From the considerations above, it is clear that the Restore phase is particularly well suited for being implemented via the stencil-reduce pattern since the computation of the functional on each pixel requires accessing to a neighbourhood of the input pixel. Alternatively, the stencil phase could be implemented via other high-level patterns, but some workaround would be required in order to express the convolution-shaped computation. For example, to compute a convolution with a map pattern, the input matrix (frame) should include also the index of each value (pixel) in order to access the neighbours from the code of the elemental function.

Finally, the two-phase filter methodology naturally induces a high-level structure for the algorithm, which can be described as the successive application of two filters as described in Fig. 3. The two phases can operate in a pipeline in the case where the two-phase restoration process is used on a stream of images (e.g. in a video application). In addition, both filters can be parallelised in a data-parallel fashion.

4) Performance considerations: In a sequential setting, the Detect stage exhibits linear execution time with respect to the total number of pixels. The Restore phase shows a computational complexity of $\mathcal{O}\left(|N| \cdot n_{-}\right.$iterations $)$, where $|N|$ is the number of noisy pixels identified in the Detect phase, and n_iterations is the number of iterations required to reach one the convergence criterion. Since the set $N$ of the noisy pixels is a fraction of the whole set of all the pixels in the image, the complexity of the two phases are actually the same. However, the Detect stage - typically a simple convolution - is two-three orders of magnitude faster than a single iteration of the second stage (Restore). Moving to parallel implementations, in particular on a fully CPU deployment, a sequential Detect stage is able to sustain the throughput of the parallel Restore stage, even in a large multicore platform. Interestingly, this is in theory no longer the case if the Restore stage is run on a large multi-GPGPU setting. In this latter case, the massive computation power provided by GPGPUs in pure data-parallel computations could reduce the execution time of the Restore phase enough to make the execution times of the two phases actually comparable. Despite of the fact we could not observe directly the described phenomenon, we can argue that a reasonably large multi-GPGPU deployment could benefit from a parallel implementation (e.g. based on stencil-reduce pattern) of the Detect phase. 


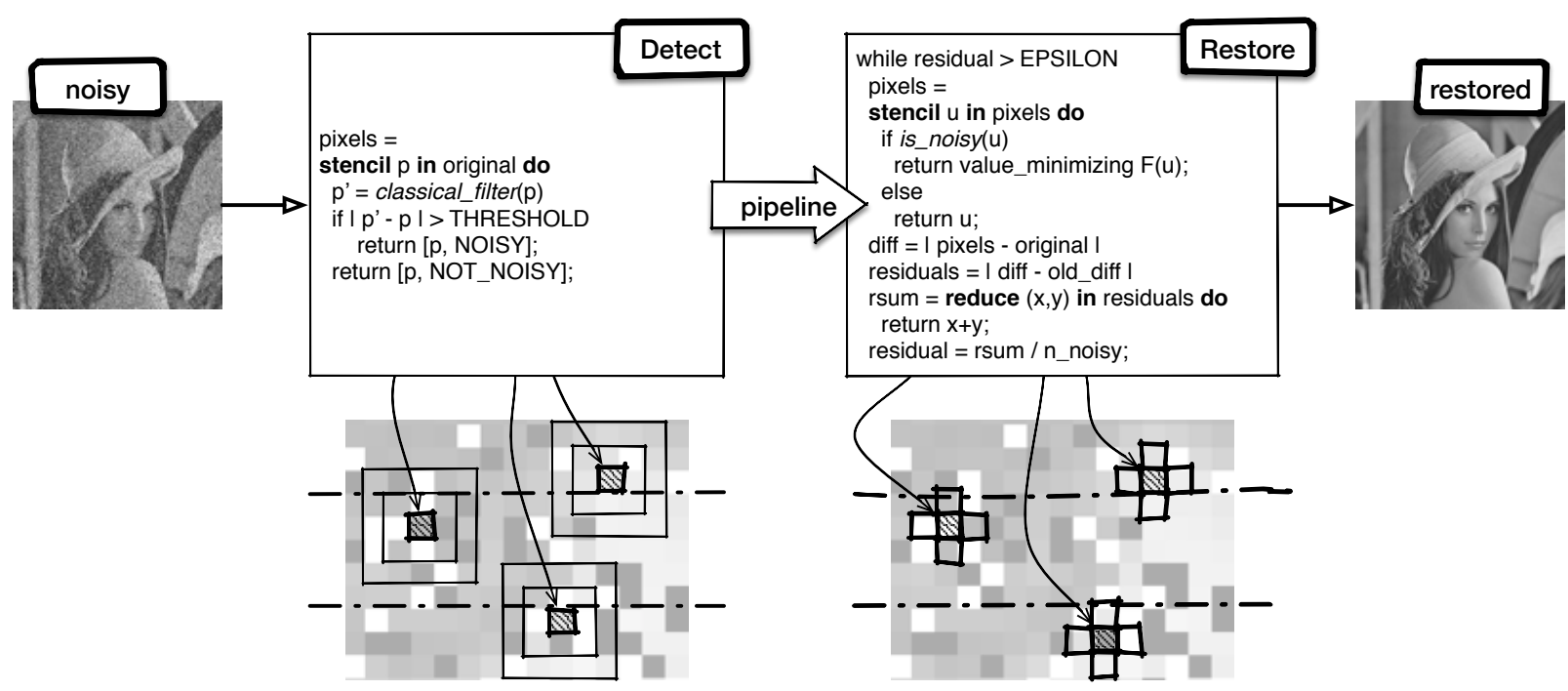

Fig. 3. Two-phase restoration for visual data streams.

\section{EXPERIMENTAL EVALUATION}

This section first shows the image restoration performance of the proposed method for Salt and Pepper and Gaussian noise on still images; second, the performance of our parallelisation strategy is discussed when employing the proposed restoration process on sample video sequences (i.e. visual data streams).

\section{Image Restoration Quality}

Among the commonly tested $256 \times 256$ 8-bit grey-scale images, Lena, Baboon and Cameraman were selected for our simulations. In case of Salt and Pepper noise, images were corrupted by "salt" (with value 255) and "pepper" (with value 0) noise with equal probability and noise levels varying from $10 \%$ to $90 \%$ with increments of $20 \%$ were tested. For Gaussian noise, we tested the restoration process performance as the variance of the noise increases from 1 to 100. The values of $\alpha$ in the regularisation function (see previous section) were set to 1.2 and $10^{-4}$ for, respectively, Salt and Pepper and Gaussian noise.

Restoration performance was measured quantitatively by the peak signal-to-noise ratio (PSNR) and the mean absolute error (MAE) defined as follows:

$$
\mathrm{PSNR}=10 \cdot \log _{10} \frac{255^{2}}{\frac{1}{M N} \sum_{i, j}\left(u_{i, j}-d_{i, j}\right)^{2}} \quad \mathrm{MAE}=\frac{1}{M N} \sum_{i, j}\left(u_{i, j}-d_{i, j}\right)^{2}
$$

with $M$ and $N$ being the image length and width and $u_{i, j}$ and $d_{i, j}$ the pixel values of the restored image and the original noisy image, respectively. Table 1 shows the obtained results.

\begin{tabular}{|c|c|c|c|c|c|c|c|c|c|c|c|c|c|c|c|c|c|c|c|c|}
\hline & \multicolumn{10}{|c|}{ Salt and Pepper } & \multicolumn{10}{|c|}{ Gaussian } \\
\hline & \multicolumn{5}{|c|}{ Lena - Noise level } & \multicolumn{5}{|c|}{ Baboon - Noise level } & \multicolumn{5}{|c|}{ Lena - Noise level (var.) } & \multicolumn{5}{|c|}{ Cameraman - Noise level (var.) } \\
\hline & $10 \%$ & $30 \%$ & $50 \%$ & $70 \%$ & $90 \%$ & $10 \%$ & $30 \%$ & $50 \%$ & $70 \%$ & $90 \%$ & 1 & 10 & 30 & 70 & 100 & 1 & 10 & 30 & 70 & 100 \\
\hline PSNR & 42.43 & 36.38 & 32.76 & 29.27 & 21.55 & 34.94 & 28.70 & 25.37 & 22.76 & 19.47 & 38.70 & 30.08 & 26.67 & 19.90 & 17.36 & 41.25 & 29.87 & 26.69 & 21.11 & 18.87 \\
\hline MAE & 0.40 & 1.38 & 2.68 & 4.82 & 15.07 & 0.92 & 3.30 & 6.43 & 10.66 & 19.78 & 1.64 & 5.25 & 8.17 & 17.32 & 24.33 & 0.90 & 4.49 & 8.11 & 15.53 & 20.16 \\
\hline
\end{tabular}

Table 1. PSNR and MAE obtained at varying of the noise affecting Lena, Baboon, and Cameraman images (added Gaussian noise has mean 0 in both cases).

To better estimate the achieved restoration quality, Fig. 4 shows the restored Baboon images as the percentage of Salt and Pepper noise increases. Fig. 5 reports, instead, the restored Cameraman images with different levels (different values of variance) of Gaussian noise. Although the performance of the proposed method is superior in the case of Salt and Pepper noise, it has to be noticed that the proposed approach also sensibly outperforms the common-adopted baseline filter for Gaussian 

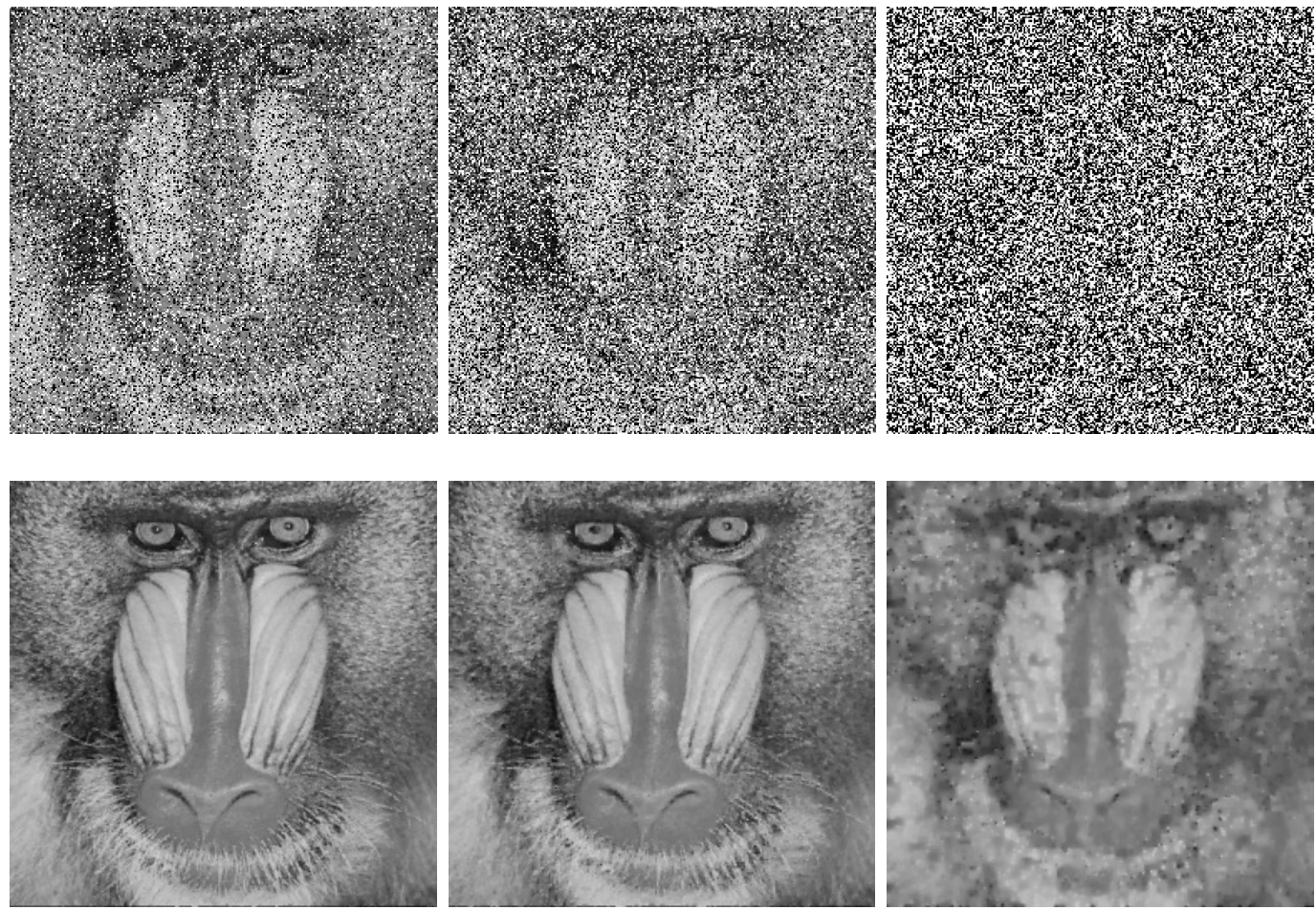

Fig. 4. First row: Baboon image affected by 30\%, 50\% and 90\% of Salt and Pepper noise. Second row: Restoration results.

noise removal, i.e. the Wiener filter, as shown in Fig. 6. We decided to use the Wiener filter as baseline since it is able to deal with pure Gaussian noise, while other existing methods (see the Background Section) operate with combinations of noises [20].

\section{Performance on Images and Video Sequences}

This section presents the obtained performance of the new visual data restoration process, implemented using the FastFlow stencil-reduce pattern. The performance has been evaluated on two different environments and compared with the multicore version (also implemented using FastFlow):

1) 16-core-HT $+2 \times$ NVidia-M2090 platform. Intel workstation with 2 eight-core double-context Xeon E5-2660 @2.2GHz, 20MB L3 shared cache, 256K L2, 64 GBytes of main memory and 2 NVidia Tesla M2090 GPGPU, Linux x86_64 and NVidia CUDA SDK 6.0.

2) 4-core-HT + NVidia-K40 platform. Intel workstation with quad-core double-context Xeon E3-1245 v3@3.4GHz, 8MB L3 shared cache, 256K L2, 16 GBytes of main memory and a NVidia K40 GPGPU, Linux x86_64 and NVidia CUDA SDK 6.0.

\begin{tabular}{|c|c|c|c|c|c|c|c|}
\hline & & \multicolumn{3}{|c|}{ Space $(4096 \times 4096$ pixels $)$} & \multicolumn{3}{|c|}{ Baboon $(2048 \times 2048$ pixels $)$} \\
\hline & & $10 \%$ & $50 \%$ & $90 \%$ & $10 \%$ & $50 \%$ & $90 \%$ \\
\hline \multirow{5}{*}{$\begin{array}{l}\text { 4-core- } H T+ \\
\text { NVidia- } K 40 \\
\text { platform }\end{array}$} & Time (s) - 1CPU & 623.60 & 3084.36 & 5556.83 & 154.73 & 770.50 & 1385.86 \\
\hline & Time (s) - 1CPU+1GPGPU & 4.55 & 17.03 & 28.73 & 1.22 & 4.33 & 7.26 \\
\hline & Speedup vs sequential - 1CPU+1GPGPU vs $1 \mathrm{CPU}$ & 137.05 & 181.15 & 193.41 & 126.73 & 178.02 & 190.90 \\
\hline & PSNR & 30.30 & 30.27 & 19.54 & 51.62 & 36.97 & 22.10 \\
\hline & MAE & 0.32 & 2.34 & 20.49 & 0.13 & 1.63 & 14.37 \\
\hline
\end{tabular}

Table 2. Performance results for restoring two sample images corrupted with Salt and Pepper noise: Space $(4096 \times 4096$ pixels $)$ and Baboon $(2048 \times 2048$ pixels), using the multicore version (with 1 denoiser thread) and the version built upon the new stencil-reduce pattern. " $1 C P U$ " stands for the execution time using only one Restore thread and the other pipeline stages (input/output operations and noise detection) are performed in parallel. 

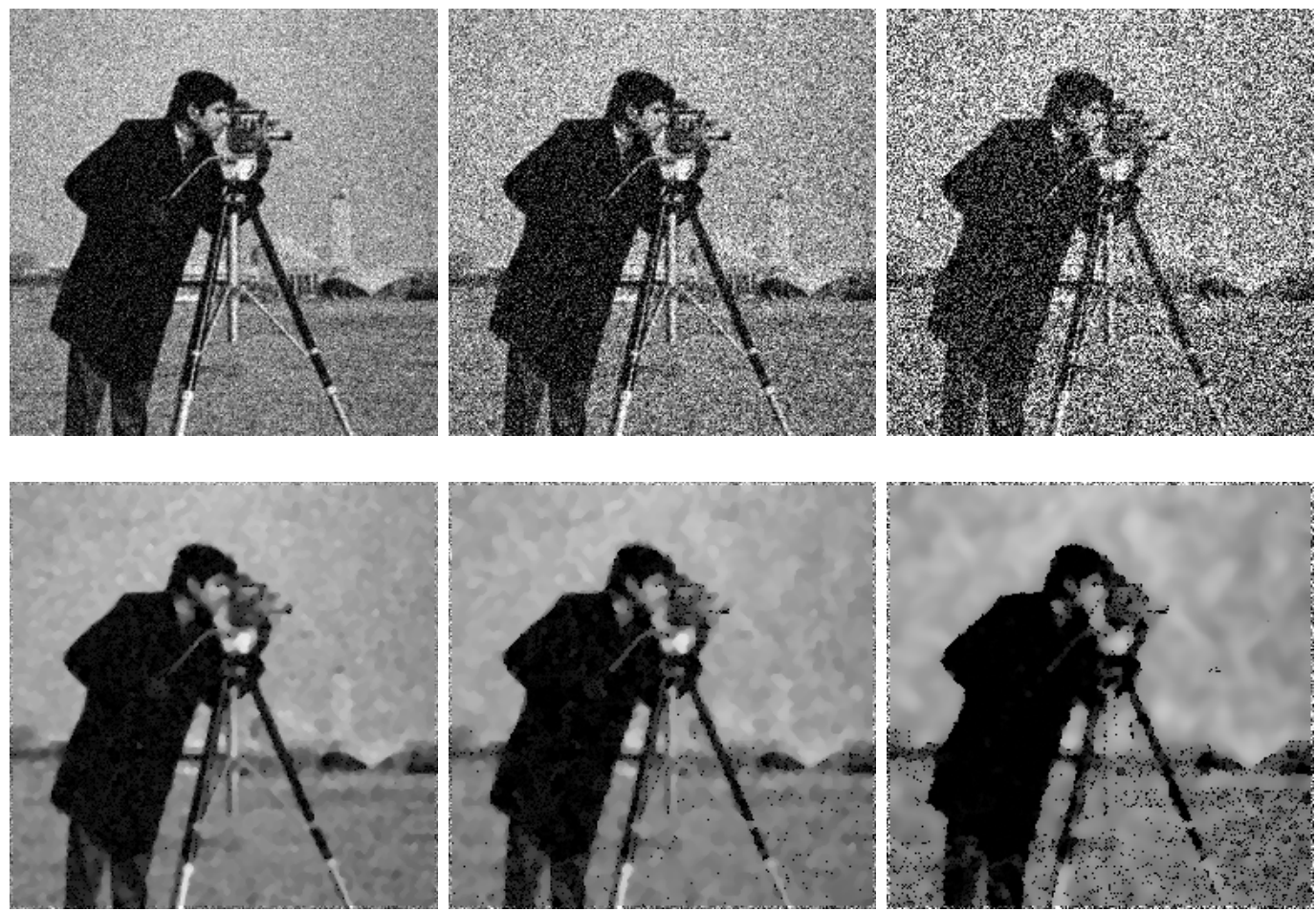

Fig. 5. First row: Cameraman image affected by Gaussian noise with variances 30, 50 and 100. Second row: Restoration results.
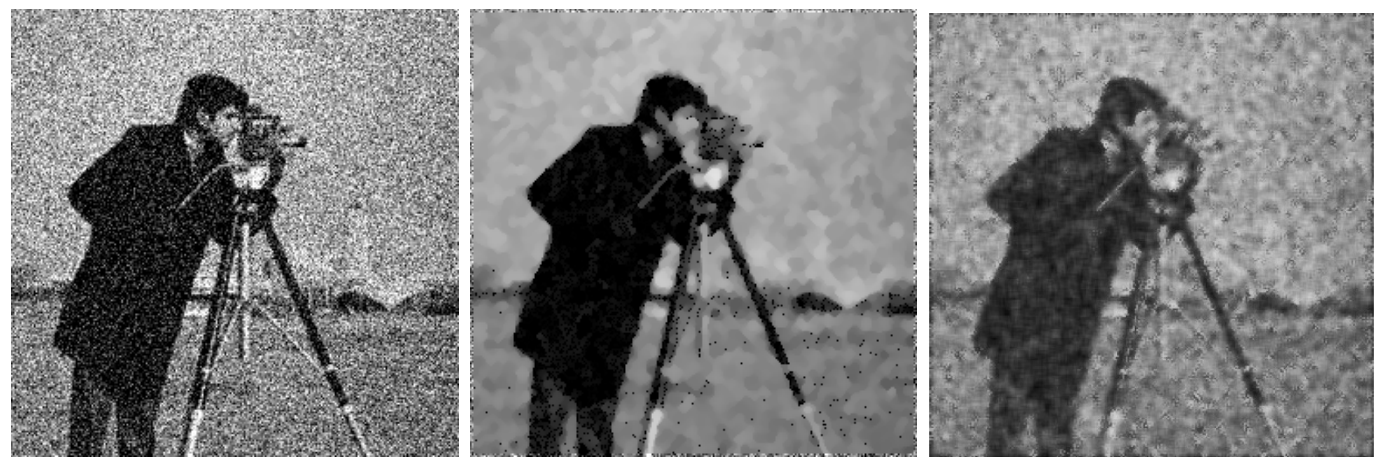

Fig. 6. Cameraman image affected by Gaussian noise with variance 50 (left image), restored with our filter (middle image) and restored with the Wiener filter using $5 \times 5$ neighbours (right image). The values of PSNR and MAE of our method are, respectively, 18.87 and 20.16, while the ones achieved by the Wiener filter are, respectively, 12.05 and 63.65 .

Table 2 presents the obtained execution times when restoring images corrupted with Salt and Pepper noise using the new FastFlow stencil-reduce GPGPU pattern, and the respective speedup comparing with the single-thread denoiser on CPU. The results present consistent speed improvements with all noise levels, but the GPGPU version is clearly faster when dealing with highly corrupted images.

Figure 7 presents the multicore (CPU-only) execution times of the video denoiser on 16-core-HT $+2 \times N$ Vidia-M2090 platform. On the left (Salt and Pepper noise), the curves show that the amount of noise directly affects the execution time. On the right, however, the curves follow the same pattern, which is explained by the fact that the added Gaussian noise affects the entire image and the detection stage uses a more sensitive threshold and thus the number pixels selected as noisy are not strongly related to the variance in these three cases. The obtained speedup, using 16 physical HT cores of the Salt and Pepper denoiser varies from $13.2 \times$ to $13.8 \times$, while the Gaussian denoisee reaches a close-to-linear speedup of $15.8 \times$.

Table 3 presents an overview of the obtained execution times and speedup on the two chosen execution environments. speedup vs multicore (1CPU+2GPGPUs vs 16CPUs and 1CPU+1GPGPU vs 4CPUs) represents the performance gain of the GPGPU version over the multicore version using all the available cores, whereas speedup vs sequential (1CPU+2GPGPUs vs 

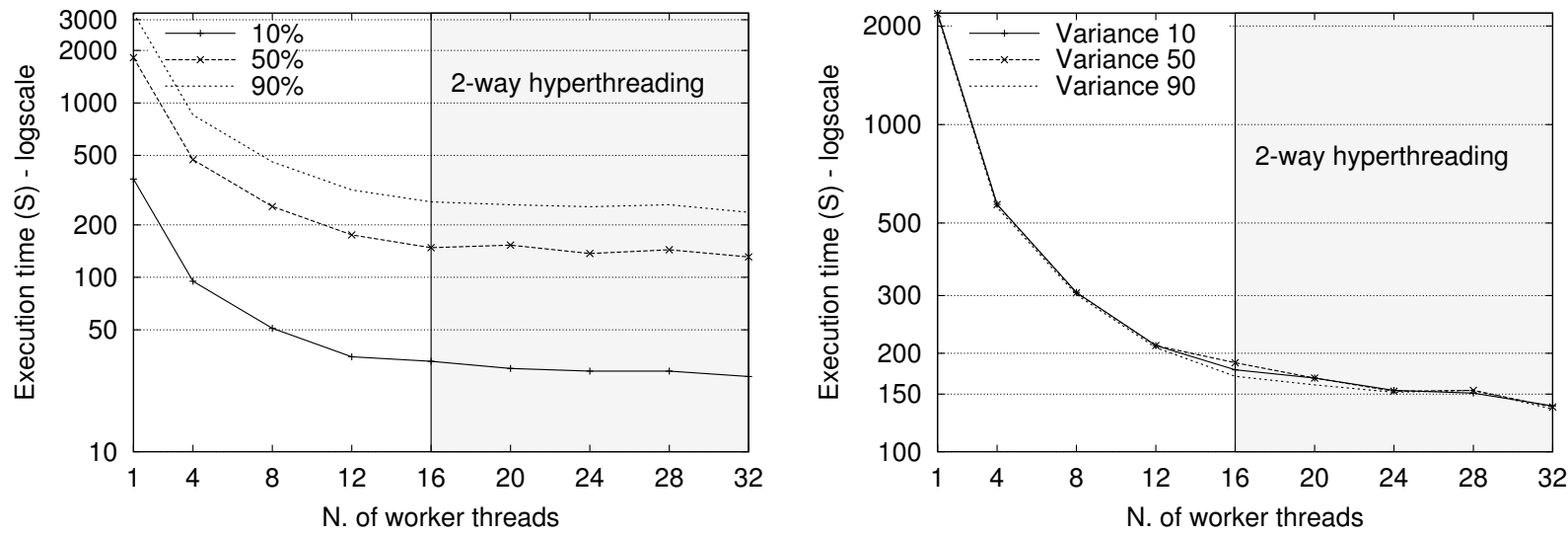

Fig. 7. Overall denoiser execution time (multicore) of a video containing 91 frames $(768 \times 512$ pixels): on the left $10 \%$, $50 \%$ and $90 \%$ of Salt and Pepper noise, on the right Gaussian noise with variance 10, 50 and 90 (and mean 0).

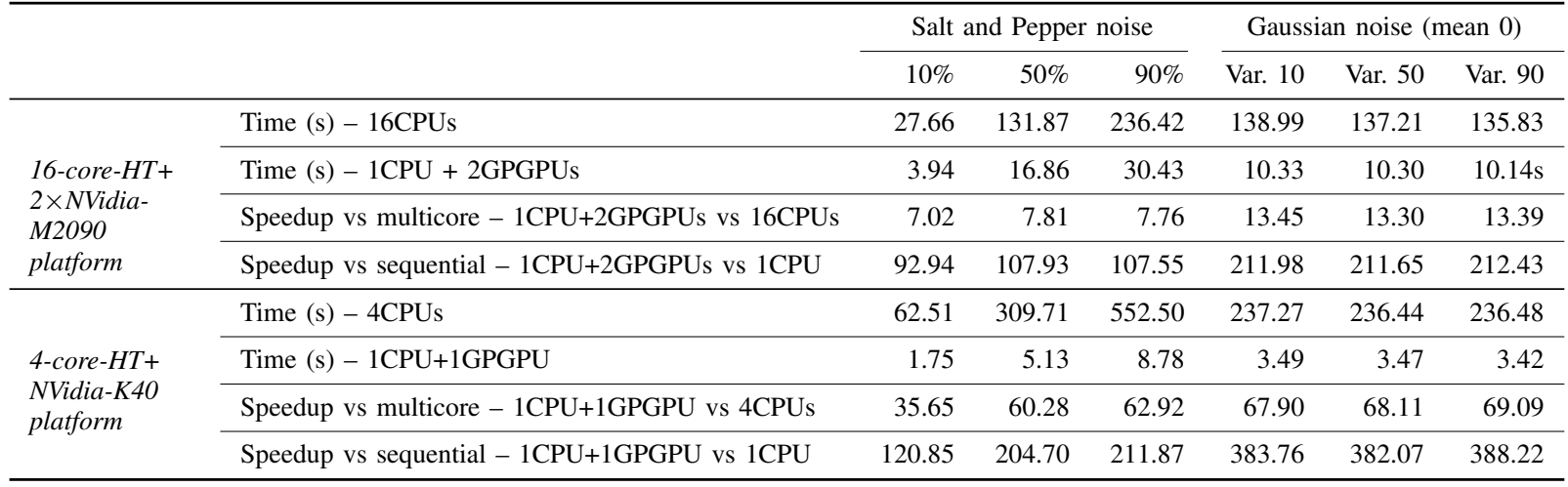

Table 3. Performance results for restoring a video containing 91 frames $(768 \times 512$ pixels $)$ on two environments, using the multicore version and the version built upon the new stencil-reduce pattern. " $1 C P U$ " stands for the execution time using only one thread for Restore stage and the other pipeline stages (input/output operations and noise detection) are performed in parallel.

$1 \mathrm{CPU}$ and 1CPU+1GPGPU vs 1CPU) represents the gain over a version that uses only one thread for Restore stage. Since it is much longer compared with the other stages on the pipeline, this version can be considered as an upper bound for the sequential performance.

The filter has been also tested with a full length movie: a one-hour video containing 107760 frames of $400 \times 304$ pixels (30fps), corrupted with $10 \%, 50 \%$ and $90 \%$ of impulse noise. This video has been restored using a NVidia K40 GPGPU and the obtained execution times are 1446s, 2622s and 6424s respectively (yielding an average throughput of 75.52fps, 41.08fps and 16.77fps).

\section{CONCLUDING REMARKS}

In this work we presented a parallel visual data restoration tool built upon a high level stencil-reduce pattern that allows to easily and efficiently program iterative applications of non-linear filters on GPGPUs. The key results here are (1) the ease by which the user can write CUDA-enabled kernels, as the framework is in charge of memory management and offloading; and (2) the obtained high performance and GPGPU occupancy (including multi-GPGPU environments). Previous work had already presented excellent performance results when restoring images affected by Salt and Pepper noise, while this work introduces a new functional for restoring Gaussian noise. Considering the good speedup and scalability preservation, we can conclude that the overhead generated by this mechanism is very low for this kind of (iterative) applications [5].

Moreover, the extension of the skeleton framework FastFlow to GPGPUs allowed us to seamlessly migrate the vision processing system to a heterogeneous setting and easily experiment with various assignments of application components to CPU/GPGPU devices. 
From a machine vision perspective, the resulting application has succeeded to demonstrate the usability of variational restoration process, considered very effective but slow, also for real-time video streams. The experiments conducted have also highlighted several issues in developing a unified parallel programming framework for heterogeneous platforms that are worth further investigation (such as the ability of offloading OpenCL kernels using the new skeleton). Thus they can steer further research in the area of parallel programming models for heterogeneous platforms.

\section{ACKNOWLEDGEMENT}

This work has been supported by the EU FP7 grant IST-2011-288570 "ParaPhrase: Parallel Patterns for Adaptive Heterogeneous Multicore Systems", Compagnia di San Paolo project id. ORTO11TPXK "IMPACT: Innovative Methods for Particle Colliders at the Terascale", and by the NVidia CUDA research center programme 2013-2015.

\section{REFERENCES}

[1] M. Nikolova, "A variational approach to remove outliers and impulse noise," Journal of Mathematical Imaging and Vision, vol. 20 , no. 1, 2004.

[2] R. Chan, C. Ho, and M. Nikolova, "Salt-and-pepper noise removal by median-type noise detectors and detail-preserving regularization," IEEE Trans. on Image Processing, vol. 14, October 2005.

[3] M. Aldinucci, C. Spampinato, M. Drocco, M. Torquati, and S. Palazzo, "A parallel edge preserving algorithm for salt and pepper image denoising," in Proc of 2nd Intl. Conference on Image Processing Theory Tools and Applications (IPTA) (K. Djemal, M. Deriche, W. Puech, and O. N. Ucan, eds.), (Istambul, Turkey), pp. 97-102, IEEE, oct 2012.

[4] M. Cole, Algorithmic Skeletons: Structured Management of Parallel Computations. Research Monographs in Par. and Distrib. Computing, Pitman, 1989.

[5] M. Aldinucci, G. Peretti Pezzi, M. Drocco, F. Tordini, P. Kilpatrick, and M. Torquati, "Parallel video denoising on heterogeneous platforms," in Proc. of Intl. Workshop on High-level Programming for Heterogeneous and Hierarchical Parallel Systems (HLPGPU), 2014.

[6] J. Astola and P. Kuosmanen, Fundamentals of Nonlinear Digital Filtering. Boca Raton, CRC, 1997.

[7] A. Bovik, Handbook of Image and Video Processing. Academic Press, 2000.

[8] T. S. Huang, G. J. Yang, and G. Y. Tang, "A fast two-dimensional median filtering algorithm," IEEE Transactions on Acoustics, Speech, and Signal Processing, vol. 27, no. 1, 1979.

[9] P. Yang and O. Basir, "Adaptive weighted median filter using local entropy for ultrasonic image denoising," in Image and Signal Processing and Analysis, 2003. ISPA 2003. Proc. of the 3rd Intl. Symposium on, vol. 2, Sept. 2003.

[10] L. Yin, R. Yang, M. Gabbouj, and Y. Neuvo, "Weighted median filters: a tutorial," Circuits and Systems II: Analog and Digital Signal Processing, IEEE Transactions on, vol. 43, Mar 1996.

[11] T.-C. Lin, "A new adaptive center weighted median filter for suppressing impulsive noise in images," Information Sciences, vol. 177, no. 4, pp. 1073 1087, 2007.

[12] P. Wang, Computing with Words. Wiley New York, 2001.

[13] T. Chen and H. R. Wu, "Impulse noise removal by multi-state median filtering," in Acoustics, Speech, and Signal Processing, 2000. ICASSP '00. Proceedings. 2000 IEEE International Conference on, vol. 6, pp. 2183-2186 vol.4, 2000.

[14] A. Faro, D. Giordano, G. Scarciofalo, and C. Spampinato, "Bayesian networks for edge preserving salt and pepper image denoising," in Image Processing Theory, Tools and Applications, 2008. IPTA 2008. First Workshops on, Nov. 2008.

[15] J.-F. Cai, R. H. Chan, and C. Fiore, "Minimization of a detail-preserving regularization functional for impulse noise removal," J. Math. Imaging Vis., vol. 29, no. $1,2007$.

[16] J.-F. Cai, R. Chan, and M. Nikolova, "Fast two-phase image deblurring under impulse noise," Journal of Mathematical Imaging and Vision, vol. 36, 2010.

[17] S. Chen and X. Yang, "A variational method with a noise detector for impulse noise removal," in Scale Space and Variational Methods in Computer Vision, vol. 4485 of LNCS, Springer, 2007.

[18] F. Cannavò, D. Giordano, G. Nunnari, and C. Spampinato, "Variational method for image denoising by distributed genetic algorithms on grid environment," in Proc. of the 15th IEEE Intl. Workshops on Enabling Technologies: Infrastructures for Collaborative Enterprises (WETICE-2006), 2006.

[19] D. Mumford and J. Shah, "Optimal approximations by piecewise smooth functions and associated variational problems," Communications on Pure and Applied Mathematics, vol. 42, no. 5, pp. 577-685, 1989.

[20] J.-F. Cai, R. H. Chan, and M. Nikolova, "Two-phase approach for deblurring images corrupted by impulse plus gaussian noise," American Institute of Mathematical Sciences, vol. 2, no. 2, pp. 187-204, 2008.

[21] Khronos OpenCL Working Group, The OpenCL Specification, version 1.0.29, 8 December 2008.

[22] S. Ernsting and H. Kuchen, "Data parallel skeletons for gpu clusters and multi-gpu systems," in Proceedings of PARCO 2011 , IOS Press, 2011.

[23] C. Augonnet, S. Thibault, R. Namyst, and P.-A. Wacrenier, "StarPU: a unified platform for task scheduling on heterogeneous multicore architectures," Concurrency and Computation: Practice and Experience, vol. 23, no. 2, pp. 187-198, 2011.

[24] J. Enmyren and C. W. Kessler, "SkePU: a multi-backend skeleton programming library for multi-gpu systems," in Proc. of the fourth Intl. workshop on High-level parallel programming and applications, HLPP '10, (New York, NY, USA), pp. 5-14, ACM, 2010.

[25] J. A. Stratton, S. S. Stone, and W. mei W. Hwu, "MCUDA: An efficient implementation of CUDA kernels for multi-core CPUs," in Languages and Compilers for Parallel Computing, 21th Intl. Workshop (LCPC) (J. N. Amaral, ed.), vol. 5335 of LNCS, pp. 16-30, Springer, 2008.

[26] P. S. Pacheco, Parallel programming with MPI. San Francisco, CA, USA: Morgan Kaufmann Publishers Inc., 1996.

[27] I. Park, M. J. Voss, S. W. Kim, and R. Eigenmann, "Parallel programming environment for OpenMP," Scientific Programming, vol. 9, pp. 143-161, 2001.

[28] Intel Corp., Threading Building Blocks, 2014 (last accessed). http://www.threadingbuildingblocks.org/.

[29] M. Aldinucci, M. Meneghin, and M. Torquati, "Efficient Smith-Waterman on multi-core with fastflow," in Proc. of Intl. Euromicro PDP 2010: Parallel Distributed and network-based Processing (M. Danelutto, T. Gross, and J. Bourgeois, eds.), (Pisa, Italy), pp. 195-199, IEEE, feb 2010.

[30] M. Aldinucci and M. Torquati, FastFlow website, 2014. http://mc-fastflow. sourceforge.net/.

[31] M. Danelutto and M. Torquati, "Loop parallelism: a new skeleton perspective on data parallel patterns," in Proc. of Intl. Euromicro PDP 2014: Parallel Distributed and network-based Processing, (Torino, Italy), IEEE, 2014.

[32] M. Aldinucci, M. Danelutto, P. Kilpatrick, and M. Torquati, "Fastflow: high-level and efficient streaming on multi-core," in Programming Multi-core and Many-core Computing Systems (S. Pllana and F. Xhafa, eds.), Parallel and Distributed Computing, ch. 13, Wiley, Mar. 2014.

[33] M. Aldinucci, M. Danelutto, P. Kilpatrick, M. Meneghin, and M. Torquati, "Accelerating code on multi-cores with fastflow," in Proc. of 17th Intl. Euro-Par 2011 Parallel Processing (E. Jeannot, R. Namyst, and J. Roman, eds.), vol. 6853 of LNCS, (Bordeaux, France), pp. 170-181, Springer, Aug. 2011. 
[34] H. González-Vélez and M. Leyton, "A survey of algorithmic skeleton frameworks: High-level structured parallel programming enablers," Software: Practice and Experience, vol. 40, no. 12, pp. 1135-1160, 2010.

[35] K. Hammond, M. Aldinucci, C. Brown, F. Cesarini, M. Danelutto, H. González-Vélez, P. Kilpatrick, R. Keller, M. Rossbory, and G. Shainer, "The paraphrase project: Parallel patterns for adaptive heterogeneous multicore systems," in Formal Methods for Components and Objects: Intl. Symposium, FMCO 2011, Torino, Italy, October 3-5, 2011, Revised Invited Lectures (B. Beckert, F. Damiani, F. S. de Boer, and M. M. Bonsangue, eds.), vol. 7542 of LNCS, pp. 218-236, Springer, 2013.

[36] M. McCool, J. Reinders, and A. Robison, Structured Parallel Programming: Patterns for Efficient Computation. San Francisco, CA, USA: Morgan Kaufmann Publishers Inc., 1st ed., 2012.

[37] C. R. Vogel and M. E. Oman, "Fast, robust total variation-based reconstruction of noisy, blurred images," IEEE Transactions on Image Processing, vol. 7, no. 6, 1998. 